\title{
Probing local innate immune responses after mucosal immunisation
}

\author{
Lindsay J Hall, Simon Clare, Gordon Dougan
}

\begin{abstract}
Background: Intranasal immunisation is potentially a very effective route for inducing both mucosal and systemic immunity to an infectious agent.

Methods: Balb/c mice were intranasally immunised with the mucosal adjuvant heat labile toxin and the Mycobacterium tuberculosis fusion protein Ag85B-ESAT6 and early changes in innate immune responses within local mucosal tissues were examined using flow cytometry and confocal microscopy. Antigen-specific humoral and cellular immune responses were also evaluated.

Results: Intranasal immunisation induced significant changes in both number and distribution of dendritic cells, macrophages and neutrophils within the nasal-associated lymphoid tissue and cervical lymph nodes in comparison to controls as early as $5 \mathrm{~h}$ post immunisation. Immunisation also resulted in a rapid and transient increase in activation marker expression first in the nasal-associated lymphoid tissue, and then in the cervical lymph nodes. This heightened activation status was also apparent from the pro-inflammatory cytokine profiles of these innate populations. In addition we also showed increased expression and distribution of a number of different cell adhesion molecules early after intranasal immunisation within these lymphoid tissues. These observed early changes correlated with the induction of a $T_{H} 1$ type immune response.
\end{abstract}

Conclusions: These data provide insights into the complex nature of innate immune responses induced following intranasal immunisation within the upper respiratory tract, and may help clarify the concepts and provide the tools that are needed to exploit the full potential of mucosal vaccines.

\section{Background}

In recent years the nasal route for vaccination has emerged as an attractive mucosal route for inducing both local and systemic immunity and offers some important opportunities for the prophylaxis of many diseases. In addition to the generation of strong local mucosal immune responses within the respiratory tract, the nose can also act as an ideal inductive and effector site for immune responses at distal mucosal sites such as the lung, gut and vagina via the common mucosal immune system [1-3] The rational design of nasal vaccines for clinical use depends on the availability of information about the mechanisms that lead to a mucosal immune response after i.n. vaccination [4]. Unfortunately, despite its role in mucosal immunity, little is

\footnotetext{
* Correspondence: I.hall@ucc.ie
Wellcome Trust Sanger Institute, Wellcome Trust Genome Campus, Hinxton,

* Correspondence: I.hall@ucc.ie
Wellcome Trust Sanger Institute, Wellcome Trust Genome Campus, Hinxton, Cambridgeshire, CB10 1SA, UK
}

(c) 2010 Hall et al; licensee BioMed Central Ltd. This is an Open Access article distributed under the terms of the Creative Commons Attribution License (http://creativecommons.org/licenses/by/2.0), which permits unrestricted use, distribution, and reproduction in any medium, provided the original work is properly cited.

known about the immune system within the upper respiratory tract (URT).

The role of lymphoid tissues in respiratory tract defences includes antigen uptake, processing and consequent presentation for the induction of mucosal immune responses. In rodents this has been found to occur in the secondary organised lymphoid aggregate, called the nasal-associated lymphoid tissue (NALT), located at the floor of the nasal cavity $[1,5,6]$. The NALT is the first point of contact for many inhaled antigens, and consequently plays a major role in both induction and effector immune responses, which are then further amplified in the draining cervical lymph nodes (CLN) [7]. In humans, the nasopharyngeal region also contains a high density of immune competent cells similar to the NALT, most notable in the Waldeyer's ring which consists of the tonsils and adenoids [8].

In addition to the generation of adaptive immune responses, the induction of innate immunity is also 
crucial for vaccines to elicit potent antigen specific immune responses. However, despite i.n. immunisation emerging as one of the most promising mucosal routes for vaccine delivery, few studies have examined the innate immune populations recruited and consequently induced within the URT early after i.n. administration of antigen. The majority of studies looking at the NALT and CLN have focussed on the induction of antigen-specific $\mathrm{T}$ and $\mathrm{B}$ lymphocytes, and have therefore tended to examine later time-points [6,9-11] A greater understanding of innate immune processes, conducted by cells relatively unrestricted in antigen specificity, including, DC, MФ and neutrophils (PMN) is therefore required.

The impact of immunisation on the expression of mucosal homing receptors on circulating immune cells, as well as mucosal addressin cell adhesion molecule-1 (MAdCAM-1) expression on endothelium, has been rather well studied, particularly with regards to the gut $[12,13]$. Oral (intestinal) mucosal exposure to antigen seems to stimulate expression of $\alpha_{4} \beta_{7}$ integrins, which together with MAdCAM-1 mediates leukocyte homing $[14,15]$. Previous studies have shown that within both the NALT and CLN, high endothelial venules (HEVs) utilise peripheral node adressin (PNAd)-L-selectin interactions and MAdCAM- $1-\alpha_{4} \beta_{7}$ interactions for leukocyte binding, although not all HEV express MAdCAM-1 $[15,16]$. However, as yet, it is still unknown whether this homing of specific cells is mediated by altered cell adhesion molecule (CAM) expression after i.n. vaccination in the URT lymphoid tissues.

As already mentioned, stimulation of the innate immune system is known to have an important role in the progression of adaptive immunity. Thus, inclusion of molecules, such as adjuvants, which can trigger early innate immune responses involved in the generation of strong and protective adaptive immune responses, is crucial to vaccine effectiveness. This is why we have included Escherichia coli heat-labile enterotoxin (LT) as a model for a strong mucosal adjuvant in our study. LT is a well characterised adjuvant which is known to induce strong immune responses after contact with mucosal surfaces when co-administered with soluble antigens [17]. In addition to LT, we also employed the use of the M. tuberculosis fusion antigen, Ag85B-ESAT6. This particular protein has been used in numerous reports and has been shown to induce strong immune responses and importantly has already been used in several recent i.n. immunisation studies [18-20]. Thus the aim of this work is to contribute to our understanding of innate immune responses induced within both the NALT and CLN early after i.n. administration of antigen and the corresponding adaptive responses that these changes provoke.

\section{Methods}

\section{Animals}

Female Balb/c mice (5-6 weeks) from Charles River, UK were used in all animal experiments. All animals were given food and water ad libitum. Mice were sacrificed by cervical dislocation or exsanguination. Animal husbandry and experimental procedures were conducted according to the United Kingdom Animals (Scientific Procedures) Act 1986.

\section{Immunisations}

For immunisations, mice were lightly anesthetised and then i.n. immunised on day 0 with $10 \mu \mathrm{g}$ Ag85B-ESAT6 plus $1 \mu \mathrm{g} \mathrm{LT}$, or PBS (naïve mice) in a volume of 15 $\mu \mathrm{L} /$ nostril. The adjuvant $\mathrm{LT}$ was a kind gift from Novartis, Italy and purified Ag85B-ESAT6 was obtained from the Statens Serum Institute, Denmark.

\section{Immunofluorescent staining}

Mice were sacrificed at 5, 24 and $72 \mathrm{~h}$ post i.n. immunisation and their CLN and NALT removed [21]. The CLN and NALT were snap-frozen in liquid nitrogen and $6 \mu \mathrm{m}$ sections cut. Frozen sections were stained with primary $\mathrm{mAb}$ as specified in Table 1 . Hoechst was used as a nuclear counterstain.

\section{Flow cytometry}

Single cell suspensions from the NALT and CLN of individual mice were prepared to obtain a final concentration of $5 \times 10^{5}$ cells/well in blocking buffer $(1 \times \mathrm{PBS} /$ $1 \%$ BSA $/ 0.05 \%$ sodium azide $/ \%$ rat, hamster and mouse serum). To this; $50 \mu \mathrm{L}$ of each mAb dye mix was added plus $5 \mu \mathrm{L}$ of the amine-reactive viability dye ViViD (Invitrogen) to determine dead cells, with incubation in the dark at $4^{\circ} \mathrm{C}$ for $30 \mathrm{~min}$ [22]. The mAb used for flow cytometry are listed in Table 1 . Cells were washed twice with blocking buffer and finally resuspended in $200 \mu \mathrm{L}$ $1 \%$ paraformaldehyde. To perform flow cytometric analyses and measure relative fluorescence intensities a FACSAria cytometer and BD Diva software (Becton Dickinson) were used. For each mouse 20,000-200,000 events were recorded. The percentage of cells labelled with each $\mathrm{mAb}$ was calculated in comparison with cells stained with isotype control antibody. Background staining was controlled by labelled isotype controls (BD Biosciences, Invitrogen and AbD Serotec) and fluorescenceminus-one (FMO). The results represent the percentage of positively stained cells in the total cell population exceeding the background staining signal. For determination of intracellular cytokine production, cells were incubated for $6 \mathrm{~h}$ at $37^{\circ} \mathrm{C}$ with BD Activation Cocktail plus GolgiPlug or GolgiPlug alone (BD Biosciences). Cells were then washed with staining buffer and stained 
Table 1 Antibodies used in study

\begin{tabular}{|c|c|c|c|c|c|}
\hline Cell Target & Target Molecule & Clone & Isotype & Conjugate & Source \\
\hline CAM & PNAd & MECA 79 & $\lg M$ & none & BD Biosciences \\
\hline CAM & MAdCAM-1 & MECA 367 & $\lg G 2 a$ & none & BD Biosciences \\
\hline CAM & VCAM-1 & MVCAM.A (429) & $\lg G 2 a$ & none & BD Biosciences \\
\hline CAM & ICAM-1 & $3 \mathrm{E} 2$ & $\lg G 1$ & none & BD Biosciences \\
\hline DC & CD11c & HL3 & $\lg G 1$ & PE-Cy7 or none & BD Biosciences \\
\hline PMN & Ly6G & RB6-8C5 & $\lg G 2 b$ & PE or none & BD Biosciences \\
\hline$M \Phi$ & $\mathrm{F} 4 / 80$ & C1:A3-1 & $\lg G 2 b$ & none & AbD Serotec \\
\hline$M \Phi$ & $\mathrm{F} 4 / 80$ & BM8 & $\lg G 2 b$ & TRI-COLOR & Invitrogen \\
\hline PMN & CD69 & $\mathrm{H} 1.2 \mathrm{~F} 3$ & $\lg G 1$ & PE or FITC & BD Biosciences \\
\hline$D C$ and $M \Phi$ & I-A $(M H C \|)$ & AF6-120.1 & $\operatorname{lgG} 2 \mathrm{a}$ & FITC & BD Biosciences \\
\hline$D C$ and $M \Phi$ & VCAM-1 & MVCAM.A (429) & $\lg G 2 a$ & Alexa Fluor 647 & AbD Serotec \\
\hline$D C, M \Phi$ and $P M N$ & IFN- $\gamma$ & XMG1.2 & $\operatorname{lgG} 1$ & Alexa Fluor 700 & BD Biosciences \\
\hline$D C, M \Phi$ and $P M N$ & $\mathrm{IL}-10$ & JES5-16E3 & $\lg G 2 b$ & APC & BD Biosciences \\
\hline $\mathrm{DC}, \mathrm{M \Phi}$ and $\mathrm{PMN}$ & TNF & MP6-XT22 & $\lg G 1$ & PE-Cy7 & BD Biosciences \\
\hline all cells & Hamster lg & none & $\lg$ & Alexa Fluor 488 or 568 & Invitrogen \\
\hline all cells & Rat lg & none & $\lg$ & Alexa Fluor 488 or 568 & Invitrogen \\
\hline
\end{tabular}

at $4^{\circ} \mathrm{C}$ with appropriate surface mAb listed in Table 1. Cells were then fixed and saponin-permeabilised (Perm/ Fix solution, BD Biosciences) and incubated with cytokine mAb listed in Table 1 or isotypic controls. After 30 min cells were twice washed in permealisation buffer (BD Biosciences) and then analysed by flow cytometry as described above.

\section{Evaluation of antibody responses}

Serum samples from mice were obtained on day 28 post immunisation and analysed for the presence of total Ig, IgG1 and IgG2a. Briefly, ELISA plates (Nunc Maxisorp) were coated overnight at $4^{\circ} \mathrm{C}$ with $50 \mu \mathrm{L}$ of a $2 \mu \mathrm{g} / \mathrm{mL}$ solution of purified Ag85B-ESAT6 in coating buffer $(0.1 \mathrm{M}$ $\mathrm{Na}_{2} \mathrm{HPO}_{4}$ at a pH of 9) and then blocked with $3.0 \%$ BSA at room temperature for $1 \mathrm{~h}$. Serum samples were diluted in $0.1 \%$ BSA starting at 1:100. Each plate contained control wells with preimmune sera, $\mathrm{PBS}(\mathrm{pH} 7.4)$, or a known positive immune serum and incubated for $1 \mathrm{~h}$ at $37^{\circ} \mathrm{C}$. Antibodies conjugated to HRP were diluted 1:1000 and plates incubated for a further $30 \mathrm{~min}$ at $37^{\circ} \mathrm{C}$. The level of HRP associated with each well was then determined using Sigma Fast $o$-phenylenediamine dihydrochloride $(50 \mu \mathrm{L}$ per well) colorimetric substrate. The reaction was stopped after 15 min by the addition of $2.5 \mathrm{M} \mathrm{H}_{2} \mathrm{SO}_{4}$. The $\mathrm{OD}_{490}$ was determined, and the titre was expressed as the reciprocal of the dilution giving an OD of 0.2.

Lung and nasal lavages, performed post-mortem, were used to measure mucosal antibody responses. Lavages were performed using $1 \mathrm{~mL}$ of ice-cold PBS containing a cocktail of protease inhibitors (Roche), which was flushed in and out of the lungs and nasal passages with a fine-tipped Pasteur pipette inserted via the trachea. The ELISA protocol was modified as follows; lavage fluid was diluted in $0.1 \%$ BSA starting at 1:10. IgA conjugated to biotin (BD Biosciences) was used as the secondary antibody at 1:1000. To detect the biotinconjugated antibody, $50 \mu \mathrm{L}$ of streptavidin-HRP diluted at 1:1000 was added to each well. Plates were developed and titres measured as described above.

\section{Cytometric Bead Array cytokine analysis}

Spleens were removed from mice 28 days post immunisation and seeded, in duplicate at a concentration of $5 \times 10^{5}$ cells/well. Splenocytes were stimulated with either Ag85BESAT6 or Concanavalin A (both at $5 \mu \mathrm{g} / \mathrm{mL}$ ) or $\mathrm{RPMI}^{+}$ medium. Plates were incubated at $37^{\circ} \mathrm{C}$ and $5 \% \mathrm{CO}_{2}$ for 36-48 h. Cytokines IL-2, IL-6, IL-12, IL-10, IFN- $\gamma$ and TNF- $\alpha$ were analysed using cytometric bead analysis flexikits (BD Biosciences) and assays were performed per the manufacturer's instructions. Samples were then analysed on a FACSAria flow cytometer (BD Biosciences). The limit of detection was $\sim 8 \mathrm{pg} / \mathrm{mL}$ for all cytokines.

\section{Statistical analysis}

Experimental results were plotted and analysed with Prism4 software (GraphPad Software Inc, CA, USA). Statistical significance was determined by using the Mann-Whitney $U$ test or by one-way ANOVA followed by Bonferroni's multiple comparison test compared to naïve mice (i.e. $0 \mathrm{~h}$ ).

\section{Results}

i.n. immunisation induces significant changes in the percentage and total cell number of innate cell populations within both the NALT and CLN

To determine what innate cells were recruited and activated after i.n. immunisation groups of mice were i.n. 
immunised with either Ag85B-ESAT6 from M. tuberculosis mixed with E. coli LT adjuvant, or PBS (naïve mice) and their NALT and CLN were removed and analysed by flow cytometry (Fig. 1 and Table 2).

Kinetic analysis revealed that i.n. immunisation differentially influenced several cell populations during early time-points, and significant changes in percentage and relative cell number were evident as early as $5 \mathrm{~h}$ post immunisation (Fig. 1 and Table 2). For example, percentages and numbers of NALT DC (defined as CD11 ${ }^{+}$), $\mathrm{M} \Phi\left(\mathrm{F} 4 / 80^{+}\right)$and PMN (as Ly6G ${ }^{+}$), were significantly $(\mathrm{p}<0.01)$ diminished $5 \mathrm{~h}$ post immunisation. However, we then observed a steady and significant $(\mathrm{p}<0.001)$ increase in NALT innate cells from $24 \mathrm{~h}$ up until $72 \mathrm{~h}$

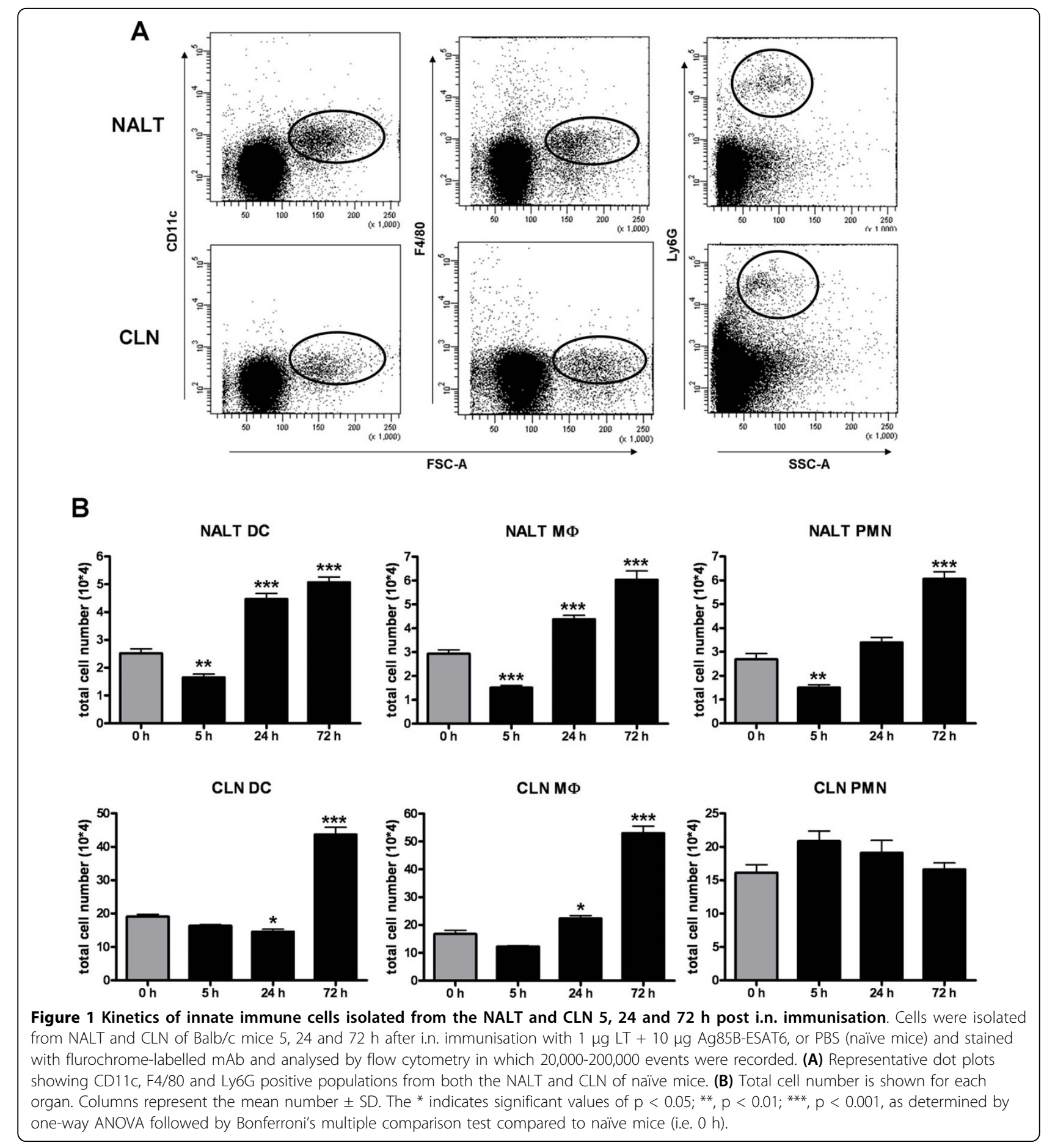


Table 2 Percentages of innate populations within the NALT and CLN after i.n. immunisation

\begin{tabular}{|c|c|c|c|c|c|}
\hline Tissue & Cell Type & $\mathrm{Oh}$ & $5 \mathrm{~h}$ & $24 \mathrm{~h}$ & $72 \mathrm{~h}$ \\
\hline \multirow[t]{3}{*}{ NALT } & DC & $6.5 \pm 0.6$ & $5.4 \pm 0.5$ & $7.8 \pm 0.8^{*}$ & $6.7 \pm 0.5$ \\
\hline & MФ & $7.6 \pm 0.8$ & $4.9 \pm 0.4^{* * *}$ & $7.6 \pm 0.9$ & $7.9 \pm 1.1$ \\
\hline & PMN & $6.9 \pm 1.1$ & $4.9 \pm 0.4^{* *}$ & $5.9 \pm 0.8$ & $8.0 \pm 1.2$ \\
\hline \multirow[t]{3}{*}{ CLN } & DC & $4.1 \pm 0.3$ & $4.3 \pm 0.3$ & $2.4 \pm 0.2^{* * *}$ & $5.5 \pm 0.6^{* * *}$ \\
\hline & $M \Phi$ & $3.6 \pm 0.3$ & $3.2 \pm 0.2$ & $3.7 \pm 0.4$ & $6.7 \pm 0.7^{* * *}$ \\
\hline & PMN & $3.5 \pm 0.3$ & $5.5 \pm 0.9^{* * *}$ & $3.2 \pm 0.3$ & $2.1 \pm 0.2^{* *}$ \\
\hline
\end{tabular}

Cells were isolated from NALT and CLN of Balb/c mice 5, 24 and $72 \mathrm{~h}$ after i.n. immunisation with PBS (naïve mice) or LT + Ag85B-ESAT6, and stained with flurochrome-labelled $\mathrm{mAb}$ and analysed by flow cytometry in which 20,000200,000 events were recorded. Numbers are mean percentage of total cells \pm SD. * indicates significant values of $\mathrm{p}<0.05 ; * *, \mathrm{p}<0.01 ; * * *, \mathrm{p}<0.001$, as determined by one-way ANOVA followed by Bonferroni's multiple comparison test compared to naïve mice (i.e. $0 \mathrm{~h}$ ).

post immunisation. The percentage of CLN PMN was increased $5 \mathrm{~h}$ post immunisation, with a subsequent reduction apparent $72 \mathrm{~h}$ post immunisation (Table 2). As observed in the NALT, total MФ numbers were also significantly $(\mathrm{p}<0.01)$ increased in the CLN of immunised mice from $24 \mathrm{~h}$. After an initial decrease in CLN $\mathrm{DC}$ population at $24 \mathrm{~h}$, this was followed by an impressive increase (over 2 times) when compared to naïve mice (Fig. 1B).

Innate populations are activated shortly after i.n. immunisation within both lymphoid tissues

i.n. immunisation also resulted in qualitative changes in both NALT and CLN innate populations during the first $72 \mathrm{~h}$ of immunisation (Fig. 2). First up-regulation of MHC II was apparent within the NALT and CLN DC and $M \Phi$ populations $5 \mathrm{~h}$ post immunisation. Likewise, VCAM-1 fluorescence was significantly increased ( $\mathrm{p}<$ 0.001 ) on DC within both tissues. Surface expression of the activation marker CD69 was increased on PMN within the NALT of immunised, compared to control mice at the $5 \mathrm{~h}$ time-point (Fig. 2). At $24 \mathrm{~h}$ we observed a significant increase $(\mathrm{p}<0.001)$ in VCAM-1 expression on both DC and MФ cells within the CLN and the NALT of immunised mice. Additionally, MHC II mean fluorescent intensity (MFI) was also significantly higher $(\mathrm{p}<0.05)$ on CLN DC and MФ. CD69 expression on PMN was again higher within the NALT of immunised mice and indeed we now also observed a significant increase $(\mathrm{p}<0.001)$ in MFI within the CLN population (Fig. 2). By $72 \mathrm{~h}$ postimmunisation only the CLN PMN population had significantly higher $(\mathrm{p}<0.001)$ surface expression of CD69 when compared to naïve mice (Fig. 2).

Innate immune populations secrete a range of cytokines shortly after i.n. immunisation

Functional aspects of the innate immune response to i.n. immunisation was addressed by ex vivo flow cytometric analysis of intracellular cytokine expression in cells from naïve and immunised animals (Fig. 3). Examining production of the anti-inflammatory cytokine IL-10; MФ and PMN NALT populations demonstrated an initial significant increase $(\mathrm{p}<0.001)$ at $5 \mathrm{~h}$ post immunisation, when compared to naïve mice, followed by a decline in percentage at $24 \mathrm{~h}$. In contrast, numbers of CLN MФ and PMN remained relatively stable during this $72 \mathrm{~h}$ period. An impressive reduction in $\mathrm{IL}-10^{+} \mathrm{DC}$ was evident in both the NALT and CLN as early as $5 \mathrm{~h}$ post immunisation and continued for the remainder of the study. The production of IFN- $\gamma$ was also examined and we observed that all innate NALT populations were significantly diminished $(\mathrm{p}<0.01) 5 \mathrm{~h}$ post immunisation. Conversely, within the CLN a dramatic increase in IFN- $\gamma^{+}$innate cells was evident when compared to stimulated naïve mice. By $24 \mathrm{~h}$ percentages of NALT IFN$\gamma^{+}$MФ and PMN were now significantly increased $(\mathrm{p}<$ 0.001) when compared to naïve animals. PMN and DC IFN- $\gamma$ producing CLN cells were also increased. At the final $72 \mathrm{~h}$ time-point NALT DC IFN- $\gamma^{+}$percentages were significantly reduced ( $\mathrm{p}<0.001$ ), but $\mathrm{M} \Phi$ numbers remained elevated. Analysis of another pro-inflammatory cytokine, TNF- $\alpha$, revealed increased production from all innate populations throughout the study when compared to similarly stimulated naïve mice. However, TNF- $\alpha$ producing PMN were significantly reduced within the CLN $72 \mathrm{~h}$ post immunisation (Fig. 3).

\section{Distribution of innate cellular populations within NALT and CLN after i.n. immunisation}

Direct in situ visualisation of both tissues revealed a similar DC and PMN distribution to that observed within the NALT of naïve mice at $5 \mathrm{~h}$ post immunisation (i.e surrounding the periphery) (Fig. $4 \mathrm{~A}$ and $4 \mathrm{C}$ ). In contrast, DC and $M \Phi$ were located more centrally (i.e. parafollicular $\mathrm{T}$ cell areas) within the CLN of immunised mice (Fig. 4A and 4B). From $24 \mathrm{~h}$ up until $72 \mathrm{~h}$ we observed a more wide-spread pattern of $D C$ and PMN populations in immunised mice compared to that seen in the naïve mice within the NALT and CLN (Fig. $4 \mathrm{~A}$ and $4 \mathrm{C}$ ). In addition, we also observed the appearance of DC within follicular regions by $72 \mathrm{~h}$. MФ present were again located centrally within both lymphoid tissues, as well as surrounding HEV in immunised mice up until the end of the study (Fig. 4B).

\section{CAM expression and distribution at early time-points within the NALT and CLN after i.n. immunisation} In order to identify the CAM expression profile early after i.n. immunisation, serial frozen NALT and CLN sections of naïve and immunised (LT + Ag85B-ESAT6) mice were investigated for the distribution and expression of MAdCAM-1, PNAd, ICAM-1 and VCAM-1 at 5, 


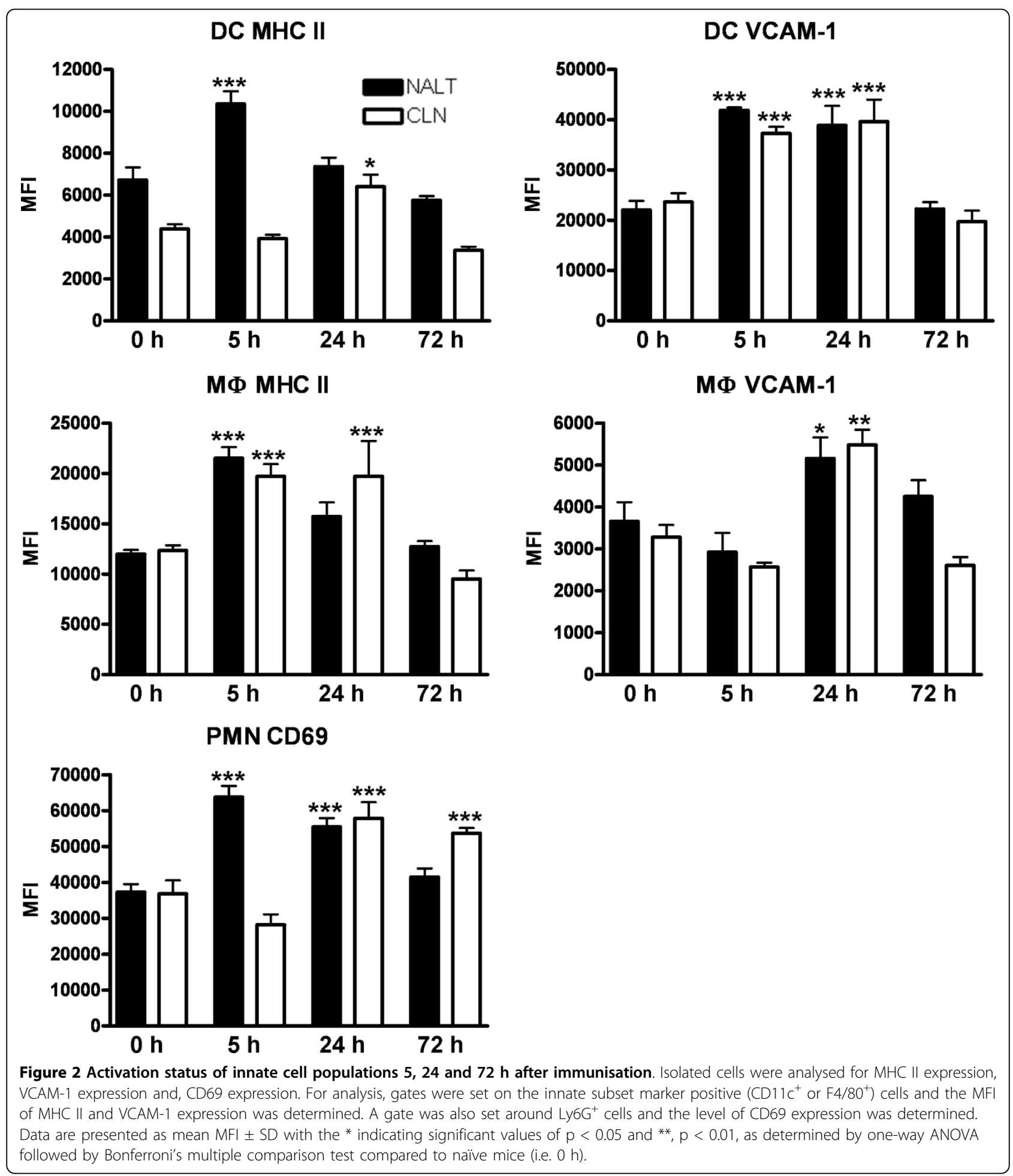

24 and 72 h. As described in previous studies, HEVs in naïve mice were found to express both MAdCAM and PNAd within their NALT and CLN (Fig. 5A and 5B) $[14,15]$. ICAM-1 was observed mainly in the vascular endothelium, and weak expression was also shown around micro-vessels within both lymphoid tissues. In addition, we also observed some cell surface ICAM-1 expression within the NALT (Fig. 5C). Weak diffuse VCAM-1 expression was observed in both tissues and was localised to the surface of cells and blood vessels, 


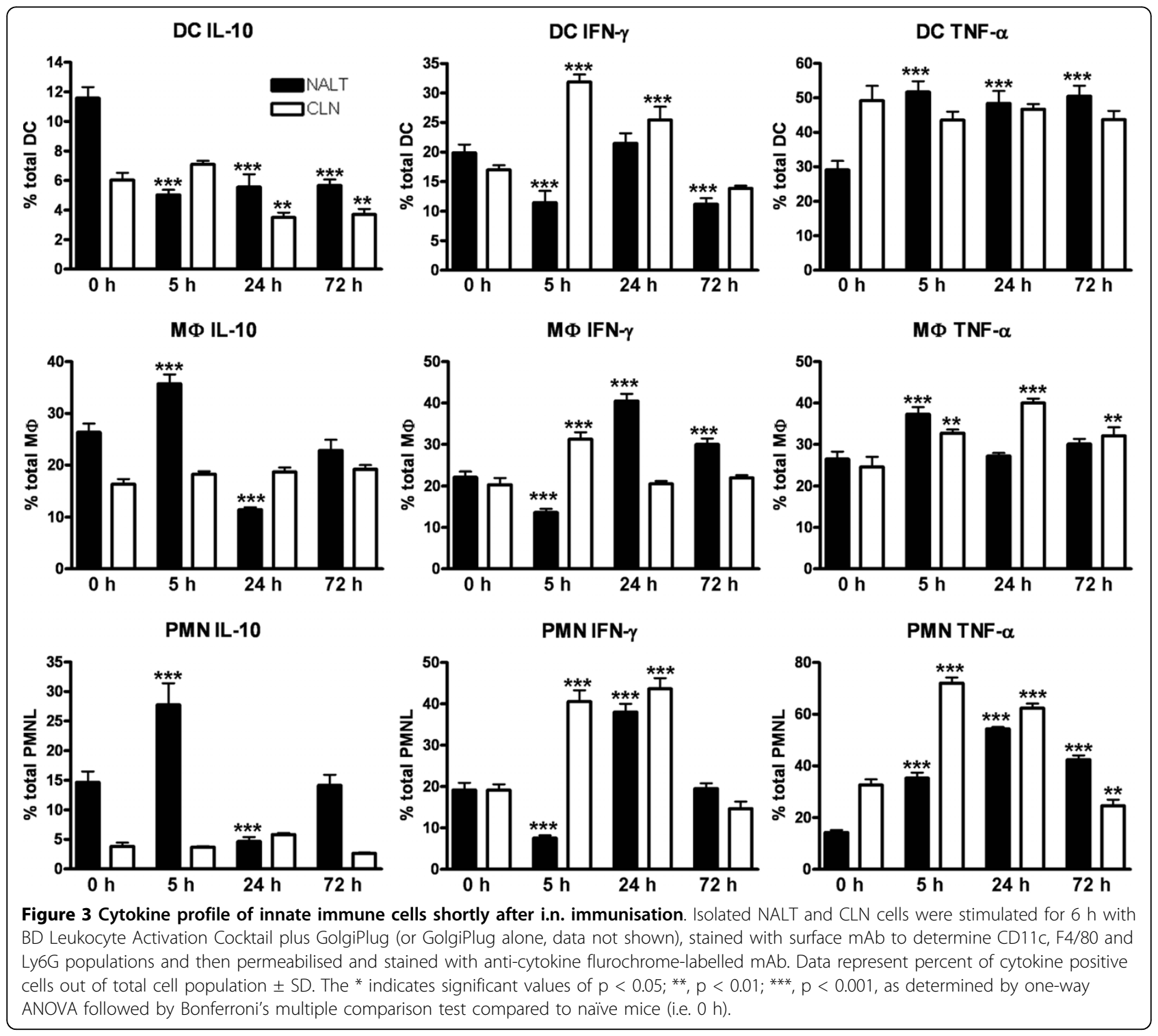

but not on HEVs within the NALT of naïve animals (Fig. 5D).

After i.n. immunisation, we visually observed a greater intensity of MAdCAM-1 expression, which was mainly located on the vascular endothelium of HEVs, and was higher than that seen in naive animals in both the NALT and CLN at all time-points (Fig. 5A). In addition to venular endothelium, MAdCAM-1 was also shown on the surface of infiltrating cells in the inflamed NALT after only $5 \mathrm{~h}$. All immunised mice were found to have some degree of diffuse MAdCAM-1 staining at all timepoints tested within the CLN. Within both tissues we observed an increase in both PNAd expression and the number of HEVs expressing this CAM as early as $5 \mathrm{~h}$ post i.n. immunisation (Fig. 5B). This expression profile was still present at $24 \mathrm{~h}$, although by $72 \mathrm{~h}$ expression levels of PNAd appeared to have reduced back to those observed in naïve animals. Greater expression of ICAM1 was observed on HEVs, micro-vessels, and with extension to many cell surfaces within the NALT and CLN of immunised mice at all time-points when compared to naïve tissues (Fig. 5C). As with ICAM-1 expression, VCAM-1 was also observed to be more intense and widespread on HEVs, blood vessels and cells in the NALT of immunised mice (Fig. 5D). Immunisation also increased VCAM-1 expression and distribution in CLN, especially at $24 \mathrm{~h}$. Expression of this CAM was also more widely distributed at $72 \mathrm{~h}$ within the CLN, but expression appeared lower than that observed at the 24 h time-point. 


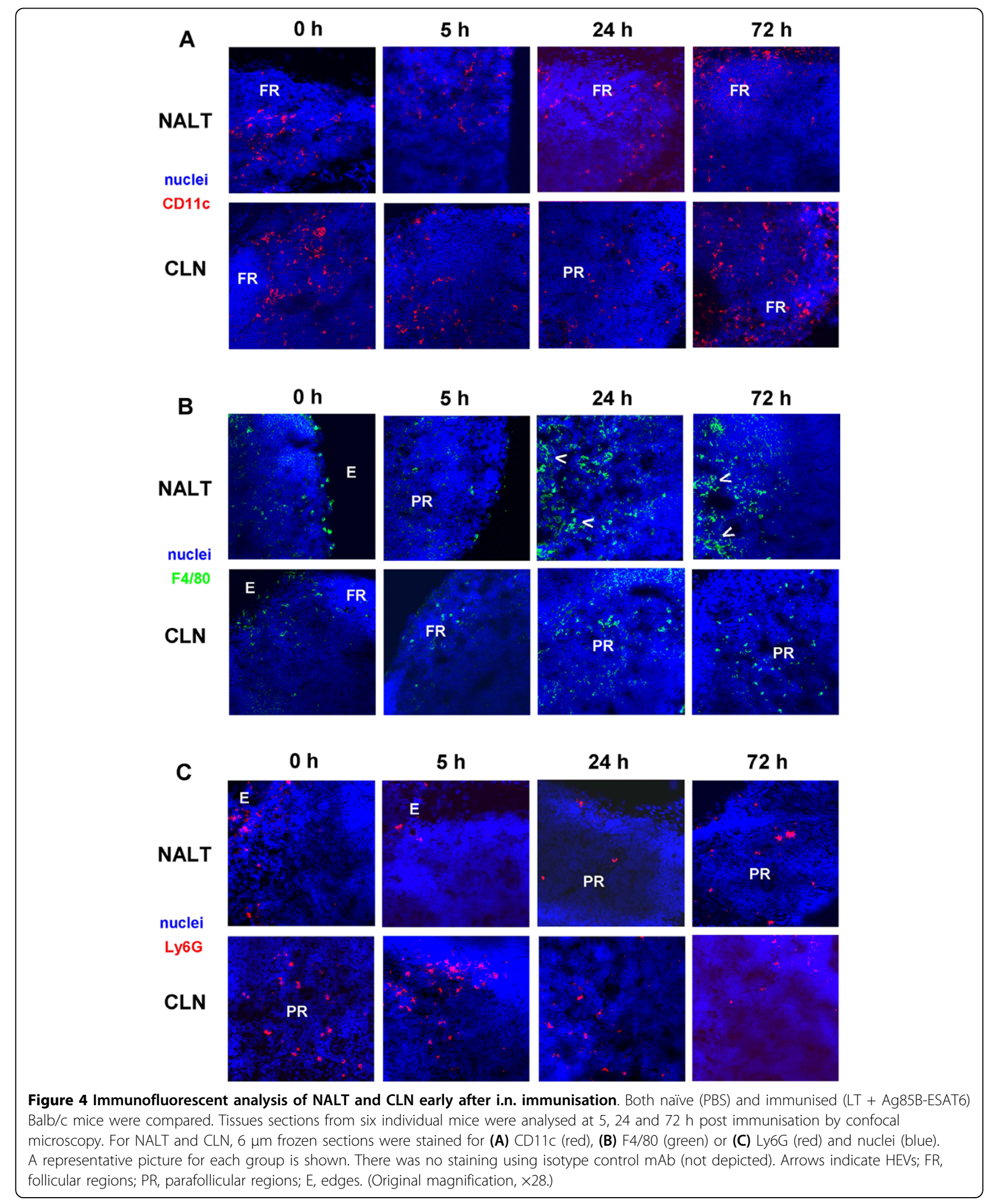




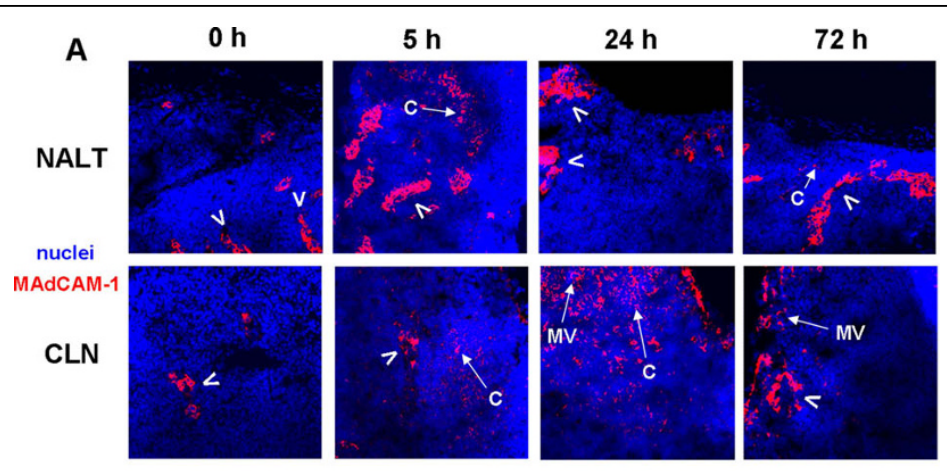

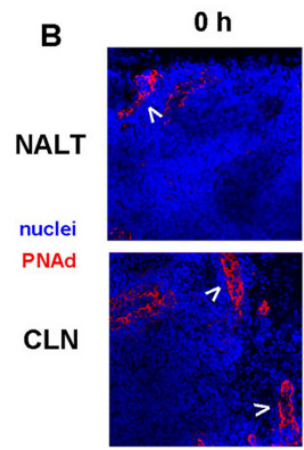

$5 \mathrm{~h}$
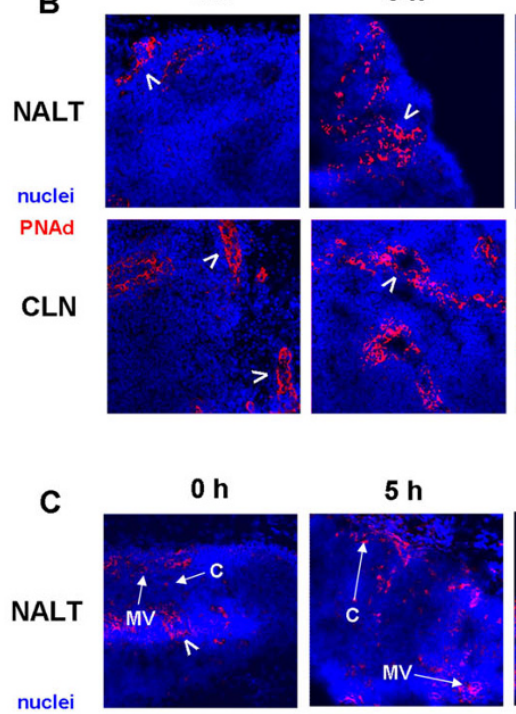

ICAM-1

CLN

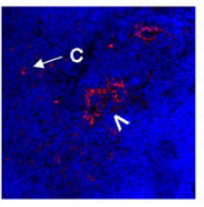

$\mathbf{O h}$

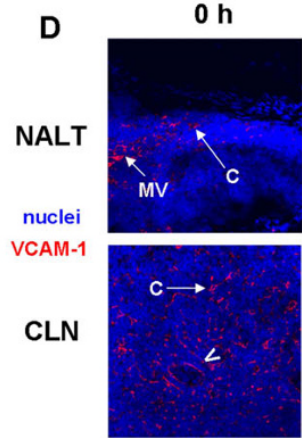

$5 \mathbf{h}$
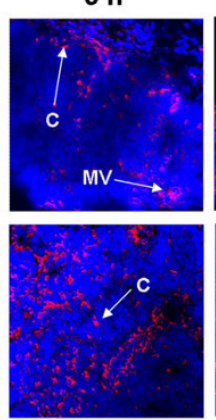

$5 \mathrm{~h}$
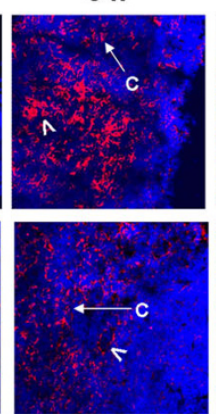

$24 \mathrm{~h}$
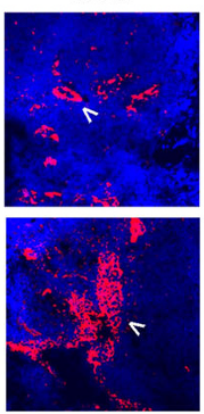

$24 \mathrm{~h}$

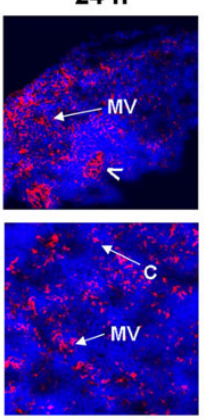

$24 \mathrm{~h}$

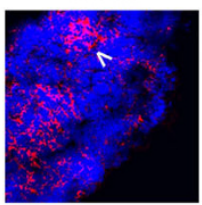

$72 \mathrm{~h}$

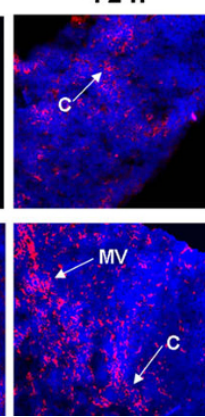

Figure 5 Differential expression of CAM in the NALT and CLN early after i.n. immunisation. Balb/c NALT and CLN were removed 5, 24 and $72 \mathrm{~h}$ post immunisation. Images represent $6 \mu \mathrm{m}$ serial frozen sections stained for (A) MAdCAM-1, (B) PNAd, (C) ICAM-1 and (D) VCAM-1 (all red) and nuclei (blue). Samples were analysed by confocal microscopy. A representative picture for each group is shown for each staining. There was no staining using isotype control mAb (not depicted). Arrows indicate HEVs; C, cells; MV, micro/blood vessel. (Original magnification, $\times 28$. 
Adaptive immune responses are induced following i.n. immunisation

In order to confirm that the changes observed in innate populations shortly after i.n. immunisation were robust enough to induce later adaptive antigen-specific immune responses we analysed both antibody and cytokine levels 28 days post immunisation (Fig. 6). Sera collected from mice immunised with LT and Ag85B-ESAT6 was observed to have significantly higher $(p<0.001)$ titres of total Ig, IgG1 and IgG2a when compared to naïve mice (Fig 6A). Additionally we also examined mucosal IgA levels and observed that immunised mice had low, but significant $(\mathrm{p}<0.05)$, titres: $122 \pm 37$ in lung and $67 \pm$ 23 in nasal washes. Spleens were also removed from immunised mice, and these cells were stimulated with Ag85B-ESAT6 in vitro to assess cytokine levels (IFN- $\gamma$, TNF- $\alpha$, IL-6, IL-10, IL-2 and IL-12). Significantly higher levels $(\mathrm{p}<0.01)$ of each cytokine were observed in LT plus Ag85B-ESAT6 immunised compared to naïve mice (Fig. 6B).

\section{Discussion}

The innate immune response reacts rapidly to foreign antigens with activation and migration of its cellular components $[23,24]$. The relevance of these individual components to host survival against pathogen infections is beyond doubt, with the protective contribution of DC, $M \Phi$, and PMN, as well as secretion of various cytokines

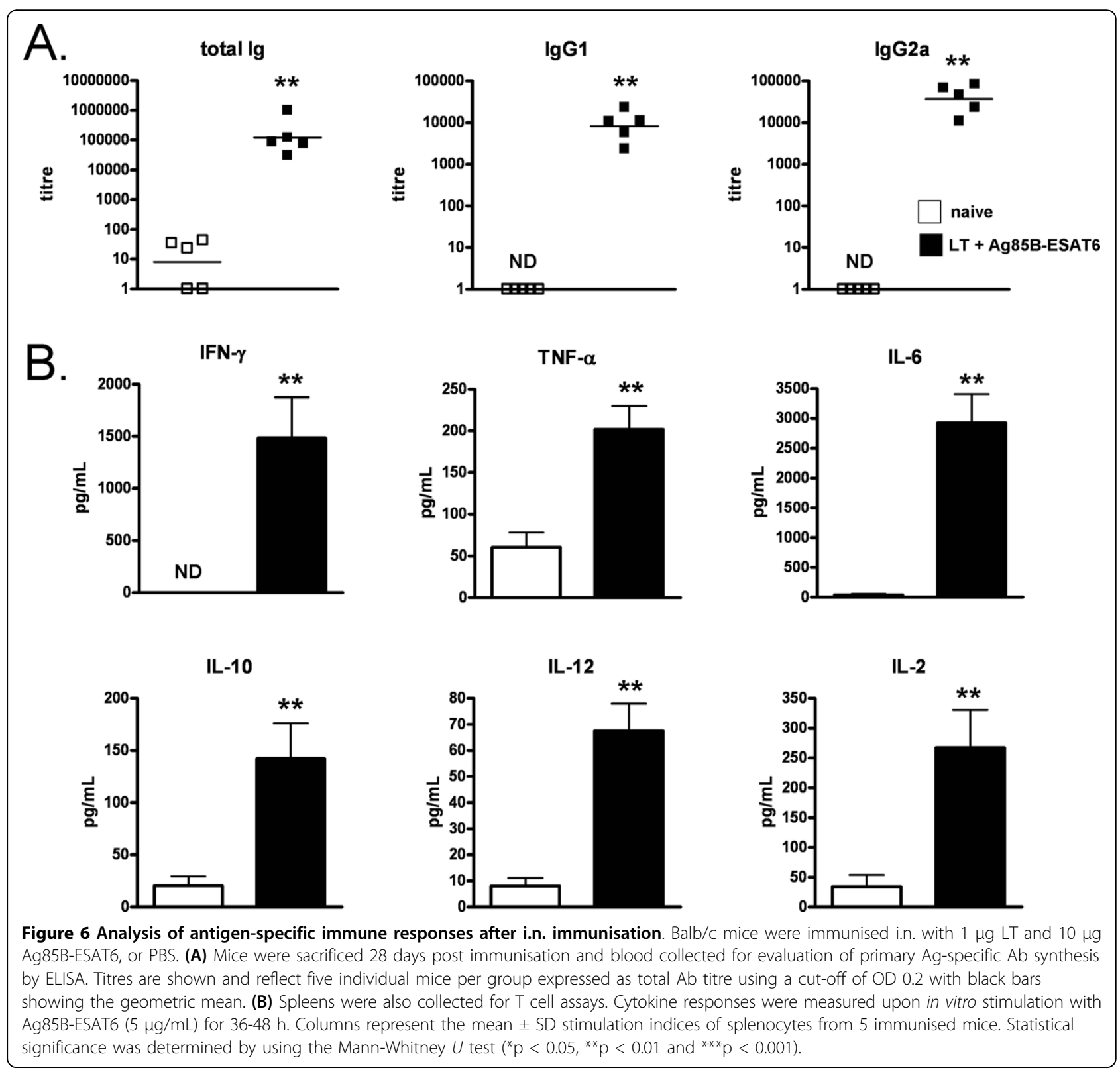


being demonstrated in many infections [25-27]. This has lead to an appreciation in recent years that innate immunity is central to protection against infectious diseases and the induction of adaptive immune responses. However, an integrated picture of innate responses induced after mucosal vaccination has not been established. This current study goes some way to addressing this by characterising changes in defined cell populations and CAM expression in the first few hours and days following i.n. immunisation with a mucosal adjuvant and model antigen and the subsequent adaptive response generated. Hopefully this study will accentuate the importance of understanding the nature of innate immune responses at the mucosae for the design of improved vaccines.

The earliest changes in NALT cell populations detected were a rapid and significant reduction in total percentages and numbers of all cell types examined. Studies to date suggest that the NALT may have an important role in the induction of mucosal immune responses after nasal immunisation, due to the large number of unswitched naïve $B$ and $T$ cells, while the nasal passages, and their associated lymphocytes, may function as an effector site [28-30]. Indeed, it may be that the cell populations undergoing changes during this early $5 \mathrm{~h}$ time-point are in fact trafficking to this area to collect and process antigen before moving back to the inductive NALT or other lymphoid tissues. All examined cells types also appeared to be activated shortly after immunisation as indicated by increases in activation marker expression. This was further confirmed when we analysed the cytokine profile of these cells. Notably, shortly after immunisation we observed a reduction in these $\mathrm{IL}-10^{+}$cells, especially within the DC population. Several studies have shown that LT toxin exerts detectable regulatory effects on DC and МФ. After LT administration, Ag-presentation was shown to be enhanced, through increased MHC class II molecule expression and peptide presentation in APC [31]. Additionally, this mucosal adjuvant can also alter the soluble immune mediator profile of these cells; increased production of IL- 6 and IL-1, but reductions in IL- 12 and NO production $[17,32]$. These findings correlate with the concurrent increase in pro-inflammatory cytokine producing cells that are essential for initiation and maintenance of later antigen specific adaptive immune responses, especially a $T_{H} 1$ response. Indeed it appears that IFN- $\gamma$ and TNF- $\alpha$ production via innate immune cells at these early time-points induces the observed robust $\mathrm{T}_{\mathrm{H}} 1$ response 28 days post immunisation. It is important to note that although activated at very early time-points, these innate cells had a similar distribution pattern when compared to naïve mice [33]. It was not until $24 \mathrm{~h}$ post immunisation that we observed movement of these cells to more central areas. This, coupled with impressive increases in number, along with their position in $\mathrm{T}$ and $\mathrm{B}$ cell areas, might reflect their involvement in immunologic reactions taking place in both these lymphoid tissues. We also observed that both $M \Phi$ and DC had up regulated expression of VCAM-1. APC expressing this CAM are known to increase presentation of antigen to germinal centre $B$ cells and localisation of APC within follicular areas suggests this subset of cells may be involved in the induction of the observed antigen-specific humoral immune responses generated after i.n. immunisation. From our staining, we also observed that these innate cells, especially $M \Phi$, were surrounding HEVs, possibly indicating migration from the URT through the lymphatic's to other lymphoid tissues for induction of further immune responses at remote sites. An overview of these cellular changes within the NALT and CLN, at all time-points, is shown in Fig. 7. The NALT is covered in ciliated respiratory epithelium interspersed with M-cells which enables the efficient absorption and sampling of antigens that are inhaled. Although not examined in this study, epithelial cells are another important cellular population that encounters foreign antigens and are notable sources of immunoregulatory cytokines and chemokines (including IL-10, TGF- $\beta$ and CXCL8). Indeed, a previous study has shown that LT enhanced IL- 6 and IL-10 secretion by intestinal epithelial cells [34]. Future studies could address the role that this cell population may play in vaccination immunity.

Another aim of this study was to examine the expression of a number of different CAM within both lymphoid tissues early after i.n. immunisation. We have shown that CAM expression of MAdCAM-1, PNAd, ICAM-1 and VCAM-1 is both increased, and more widely distributed, at all examined time-points in both the NALT and CLN of immunised mice. It has previously been demonstrated that both NALT and CLN express all these CAMs on their HEVs $[14,15,35]$. We also observed expression of these addressins on HEVs in naïve mice and in addition, we found that as early as 5 $h$ post i.n. immunisation, expression of MAdCAM-1 and PNAd was further up regulated on HEVs in both tissues. This increased expression is similar to that observed in other studies after either oral immunisation or challenge in the gut mucosa [13,36-38]. It is likely that the increased MAdCAM-1 and PNAd expression in the respiratory mucosa after immunisation, possibly due to the increased local production of pro-inflammatory cytokines via innate cells, is one of the mechanisms controlling the recruitment of leukocytes to the site of inflammation [15]. We also observed that ICAM-1 and VCAM-1 expression was dramatically induced, following i.n. immunisation, on both vascular endothelium and 


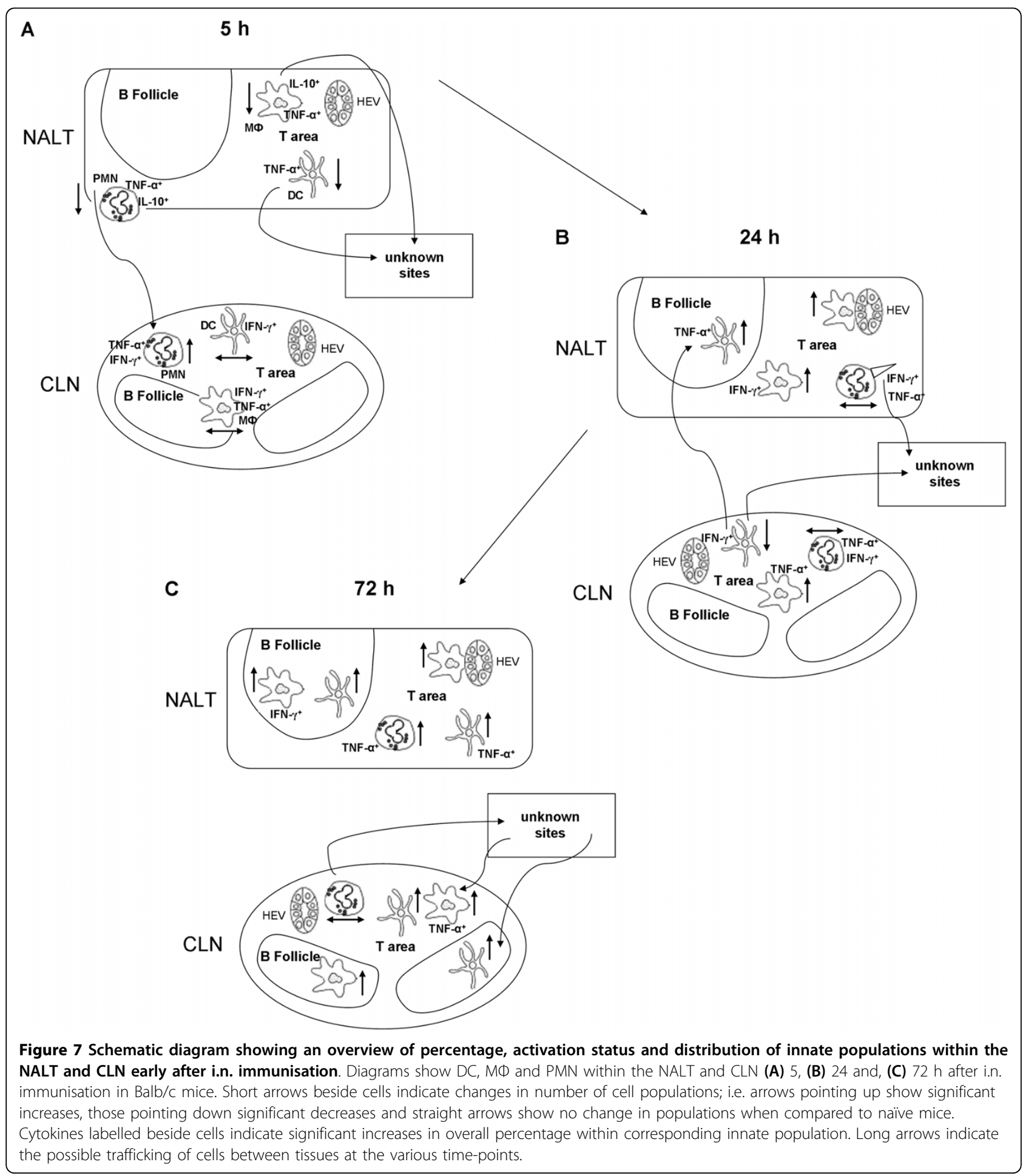

cell surfaces indicating that leukocyte trafficking to the NALT and CLN may also depend on the interactions of $\alpha_{\mathrm{L}} \beta_{2}$ and $\alpha_{4} \beta_{1}$ cells. It is interesting to note that these same molecules are involved in trafficking in the genitourinary tract, which may explain why high levels of antigen-specific immune responses are induced in the genital tract after nasal immunisation [39]. As already discussed, VCAM-1 is essential for binding of follicular $\mathrm{DC}$ to $\mathrm{B}$ cells and for the consequent formation of germinal centres. Additionally, along with ICAM-1, 
these molecules enable binding to $\mathrm{T}$ cells to form part of the stable T cell-APC immunological synapse, which is essential for antigen presentation [40-42]. The increased expression of VCAM-1 and ICAM-1 on the surface of leukocytes indicates a possible role in early recruitment and retention of immune cells, enabling increased antigen presentation, and the consequent induction of antigen specific antibody and cytokine immune responses we observed.

\section{Conclusions}

From our data it appears that specific stimulation of innate responses, through administration of adjuvant and antigen i.n., enables conditioning of the immune system for subsequent development of specific $T_{H} 1$ adaptive immunity. These findings may consequently help in the design and construction, via specific targeting of innate populations, of new vaccines against diseases that require this type of immune response for protection. It is hoped that this current research will ultimately provide researchers with a clearer and more informed platform for studying basic immune mechanisms, and may help provide the tools required to exploit the full potential of mucosal vaccines.

\section{Acknowledgements}

This study was carried out with financial support from the Commission of the European Communities, Sixth Framework Programme, contract LSHP-CT2003-503240, Mucosal Vaccines for Poverty-Related Diseases (MUVAPRED) and The Wellcome Trust. We would also like to thank Novartis, Italy and Statens Serum Institute, Denmark for their kind gifts of purified LT and Ag85B-ESAT6.

\section{Authors' contributions}

LH contributed to the design of the study, carried out all experiments and analysis and drafted the manuscript. SC participated in the design of the study, flow cytometry experiments and drafting of the manuscript. GD conceived the study, and participated in its design and helped to draft the manuscript. All authors read and approved the final manuscript.

\section{Competing interests}

The authors declare that they have no competing interests.

Received: 1 April 2010 Accepted: 13 September 2010

Published: 13 September 2010

\section{References}

1. Davis SS: Nasal Vaccines. Adv Drug Deliver Rev 2001, 51:21-42.

2. Wu HY, Russell MW: Nasal lymphoid tissue, intranasal immunization, and compartmentalization of the common mucosal immune system. Immunol Res 1997, 16(2):187-201.

3. Ouhara K, Komatsuzawa H, Shiba H, Uchida Y, Kawai T, Sayama K, Hashimoto K, Taubman MA, Kurihara H, Sugai M: Actinobacillus actinomycetemcomitans outer membrane protein 100 triggers innate immunity and production of beta-defensin and the 18-kilodalton cationic antimicrobial protein through the fibronectin-integrin pathway in human gingival epithelial cells. Infect Immun 2006, 74(9):5211-5220.

4. Ogra PL, Faden H, Welliver RC: Vaccination strategies for mucosal immune responses. Clin Microbiol Rev 2001, 14(2):430-445.

5. Kiyono H, Fukuyama S: NALT-versus Peyer's-patch-mediated mucosal immunity. Nat Rev Immunol 2004, 4(9):699-710.
6. Zuercher AW, Coffin SE, Thurnheer MC, Fundova P, Cebra JJ: Nasalassociated lymphoid tissue is a mucosal inductive site for virus-specific humoral and cellular immune responses. J Immunol 2002, 168(4):1796-1803.

7. Bienenstock J, McDermott MR: Bronchus- and nasal-associated lymphoid tissues. Immunol Rev 2005, 206:22-31.

8. Perry M, Whyte A: Immunology of the tonsils. Immunol Today 1998, 19(9):414-421.

9. Medaglini D, Ciabattini A, Cuppone AM, Costa C, Ricci S, Costalonga M, Pozzi G: In vivo activation of naïve CD4+ T cells in nasal mucosaassociated lymphoid tissue following intranasal immunization with recombinant Streptococcus gordonii. Infect Immun 2006, 74(5):2760-2766.

10. Kurono Y, Yamamoto M, Fujihashi K, Kodama S, Suzuki M, Mogi G, McGhee JR, Kiyono H: Nasal immunization induces Haemophilus influenzae-specific Th1 and Th2 responses with mucosal $\lg A$ and systemic IgG antibodies for protective immunity. J Infect Dis 1999, 180(1):122-132.

11. Wiley JA, Hogan RJ, Woodland DL, Harmsen AG: Antigen-specific CD8(+) T cells persist in the upper respiratory tract following influenza virus infection. J Immunol 2001, 167(6):3293-3299.

12. Kantele A, Zivny J, Hakkinen M, Elson CO, Mestecky J: Differential homing commitments of antigen-specific T cells after oral or parenteral immunization in humans. J Immunol 1999, 162(9):5173-5177.

13. Lindholm C, Naylor A, Johansson EL, Quiding-Jarbrink M: Mucosal vaccination increases endothelial expression of mucosal addressin cell adhesion molecule 1 in the human gastrointestinal tract. Infect Immun 2004, 72(2): 1004-1009.

14. Csencsits KL, Jutila MA, Pascual DW: Nasal-associated lymphoid tissue: Phenotypic and functional evidence for the primary role of peripheral node addressin in naïve lymphocyte adhesion to high endothelial venules in a mucosal site. J Immunol 1999, 163(3):1382-1389.

15. Csencsits $K L$, Jutila MA, Pascual DW: Mucosal addressin expression and binding-interactions with naïve lymphocytes vary among the cranial, oral, and nasal-associated lymphoid tissues. Eur J Immunol 2002, 32(11):3029-3039.

16. Wolvers DA, Coenen-de Roo CJ, Mebius RE, van der Cammen MJ, Tirion F, Miltenburg AM, Kraal G: Intranasally induced immunological tolerance is determined by characteristics of the draining lymph nodes: studies with OVA and human cartilage gp-39. J Immunol 1999, 162(4):1994-1998.

17. Freytag LC, Clements JD: Mucosal adjuvants. Vaccine 2005, 23(15):1804-1813.

18. Hou Y, Hu WG, Hirano T, Gu XX: A new intra-NALT route elicits mucosal and systemic immunity against Moraxella catarrhalis in a mouse challenge model. Vaccine 2002, 20(17-18):2375-2381.

19. Dietrich J, Andersen C, Rappuoli R, Doherty TM, Jensen CG, Andersen P: Mucosal administration of Ag85B-ESAT-6 protects against infection with Mycobacterium tuberculosis and boosts prior bacillus Calmette-Guerin immunity. J Immunol 2006, 177(9):6353-6360.

20. Andersen CS, Dietrich J, Agger EM, Lycke NY, Lovgren K, Andersen P: The Combined CTA1-DD/ISCOMs Vector Is an Effective Intranasal Adjuvant for Boosting Prior Mycobacterium bovis BCG Immunity to Mycobacterium tuberculosis. Infect Immun 2007, 75(1):408-416.

21. Asanuma H, Thompson AH, Iwasaki T, Sato $Y$, Inaba $Y$, Aizawa C, Kurata T, Tamura SI: Isolation and characterization of mouse nasal-associated lymphoid tissue. J Immunol Methods 1997, 202(2):123-131.

22. Perfetto SP, Chattopadhyay PK, Lamoreaux L, Nguyen R, Ambrozak D, Koup RA, Roederer M: Amine reactive dyes: an effective tool to discriminate live and dead cells in polychromatic flow cytometry. $J$ Immunol Methods 2006, 313(1-2):199-208.

23. Janeway CA Jr, Medzhitov R: Innate immune recognition. Annu Rev Immunol 2002, 20:197-216.

24. Medzhitov R, Janeway C Jr: Innate immunity. N Engl J Med 2000, 343(5):338-344.

25. Pedrosa J, Saunders BM, Appelberg R, Orme IM, Silva MT, Cooper AM: Neutrophils play a protective nonphagocytic role in systemic Mycobacterium tuberculosis infection of mice. Infect Immun 2000, 68(2):577-583.

26. Samsom JN, Annema A, Groeneveld PH, van Rooijen N, Langermans JA, van Furth R: Elimination of resident macrophages from the livers and spleens of immune mice impairs acquired resistance against a secondary Listeria monocytogenes infection. Infect Immun 1997, 65(3):986-993. 
27. Weissman D, Fauci AS: Role of dendritic cells in immunopathogenesis of human immunodeficiency virus infection. Clin Microbiol Rev 1997, 10(2):358-367.

28. Kuper CF, Koornstra PJ, Hameleers DMH, Biewenga J, Spit BJ, Duijvestijn AM, Vriesman P, Sminia T: The Role of Nasopharyngeal Lymphoid-Tissue. Immunol Today 1992, 13(6):219-224.

29. Hiroi T, Iwatani K, lijima H, Kodama S, Yanagita M, Kiyono H: Nasal immune system: distinctive Th0 and Th1/Th2 type environments in murine nasalassociated lymphoid tissues and nasal passage, respectively. Eur J Immunol 1998, 28(10):3346-3353.

30. Wu HY, Nikolova EB, Beagley KW, Russell MW: Induction of antibodysecreting cells and T-helper and memory cells in murine nasal lymphoid tissue. Immunology 1996, 88(4):493-500.

31. Petrovska L, Lopes L, Simmons CP, Pizza M, Dougan G, Chain BM: Modulation of dendritic cell endocytosis and antigen processing pathways by Escherichia coli heat-labile enterotoxin and mutant derivatives. Vaccine 2003, 21(13-14):1445-1454.

32. Martin M, Sharpe A, Clements JD, Michalek SM: Role of B7 costimulatory molecules in the adjuvant activity of the heat-labile enterotoxin of Escherichia coli. J Immunol 2002, 169(4):1744-1752.

33. Gueirard P, Ave P, Balazuc AM, Thiberge S, Huerre M, Milon G, Guiso N: Bordetella bronchiseptica persists in the nasal cavities of mice and triggers early delivery of dendritic cells in the lymph nodes draining the lower and upper respiratory tract. Infect Immun 2003, 71(7):4137-4143.

34. Soriani M, Bailey L, Hirst TR: Contribution of the ADP-ribosylating and receptor-binding properties of cholera-like enterotoxins in modulating cytokine secretion by human intestinal epithelial cells. Microbiology (Reading, England) 2002, 148(Pt 3):667-676.

35. Hussain I, Randolph D, Brody SL, Song SK, Hsu A, Kahn AM, Chaplin DD, Hamilos DL: Induction, distribution and modulation of upper airway allergic inflammation in mice. Clin Exp Allergy 2001, 31(7):1048-1059.

36. Quiding-Jarbrink M, Nordstrom I, Granstrom G, Kilander A, Jertborn M, Butcher EC, Lazarovits Al, Holmgren J, Czerkinsky C: Differential expression of tissue-specific adhesion molecules on human circulating antibodyforming cells after systemic, enteric, and nasal immunizations. A molecular basis for the compartmentalization of effector $B$ cell responses. J Clin Invest 1997, 99(6):1281-1286.

37. Rydstrom A, Wick MJ: Monocyte Recruitment, Activation, and Function in the Gut-Associated Lymphoid Tissue during Oral Salmonella Infection. J Immunol 2007, 178(9):5789-5801.

38. Salmi M, Granfors K, MacDermott R, Jalkanen S: Aberrant binding of lamina propria lymphocytes to vascular endothelium in inflammatory bowel diseases. Gastroenterology 1994, 106(3):596-605.

39. Perry LL, Feilzer K, Portis JL, Caldwell HD: Distinct homing pathways direct T lymphocytes to the genital and intestinal mucosae in Chlamydiainfected mice. J Immunol 1998, 160(6):2905-2914.

40. Carrasco YR, Fleire SJ, Cameron T, Dustin ML, Batista FD: LFA-1/ICAM-1 interaction lowers the threshold of $B$ cell activation by facilitating $B$ cell adhesion and synapse formation. Immunity 2004, 20(5):589-599.

41. Balogh P, Aydar Y, Tew JG, Szakal AK: Appearance and phenotype of murine follicular dendritic cells expressing VCAM-1. Anat Rec 2002, 268(2):160-168.

42. Dustin ML, Shaw AS: Costimulation: building an immunological synapse. Science 1999, 283(5402):649-650.

doi:10.1186/1476-8518-8-5

Cite this article as: Hall et al.: Probing local innate immune responses after mucosal immunisation. Journal of Immune Based Therapies and Vaccines 2010 8:5.

\section{Submit your next manuscript to BioMed Central and take full advantage of:}

- Convenient online submission

- Thorough peer review

- No space constraints or color figure charges

- Immediate publication on acceptance

- Inclusion in PubMed, CAS, Scopus and Google Scholar

- Research which is freely available for redistribution

Submit your manuscript at www.biomedcentral.com/submit 